\title{
TEST MODULES FOR PROJECTIVITY
}

\author{
P. JOTHILINGAM
}

\begin{abstract}
Let $R$ be a commutative noetherian local ring with identity. Modules over $R$ will be assumed to be finitely generated and unitary. A nonzero $R$-module $M$ is said to be a strong test module for projectivity if the condition $\operatorname{Ext}_{R}^{1}(P, M)=(0)$, for an arbitrary module $P$. implies that $P$ is projective. This definition is due to Mark Ramras [5]. He proves that a necessary condition for $M$ to be a strong test module is that depth $M \leqslant 1$. This is also easy to see. In this note it is proved that, over a regular local ring, this condition is also sufficient for $M$ to qualify as a strong test module.
\end{abstract}

By $R$ we mean a commutative noetherian local ring with identity. Module means a finitely generated unitary $R$-module. We use [6] as a standing reference for unexplained terms and basic facts on local algebra.

In [5], Mark Ramras introduced the notion of strong test module.

Definition. Let $M$ be a nonzero $R$-module. $M$ is said to be a strong test module if $\operatorname{Ext}_{R}^{1}(P, M)=(0)$, for an arbitrary module $P$, implies that $P$ is projective.

It is known that the residue field and the maximal ideal of $R$ are examples of strong test modules. Besides, a necessary condition for $M$ to be a strong test module is that depth $M \leqslant 1$. This is well known and easy [5]. It is the purpose of this note to show that over a regular local ring this condition is also sufficient for $M$ to qualify as a strong test module. This is Theorem 1. The proof depends on several lemmas which are well known. As usual * denotes duals and p.d. stands for projective dimension.

Lemma 1. Suppose $A$ and $B$ are nonzero $R$-modules. Let p.d. $A=n$. Then $\operatorname{Ext}_{R}^{n}(A, B) \neq(0)$.

ProOf. See [2].

LEMma 2. Let $A$ and $B$ be modules over the regular local ring $R, B \neq(0)$. If $\operatorname{Ext}_{R}^{1}(A, B)=(0)$, then $\operatorname{Ext}_{R}^{1}(A, R)=(0)$ and the natural map $A^{*} \otimes B \rightarrow$ $\operatorname{Hom}(A, B)$ is an isomorphism.

Proof. See [3].

We also need a fundamental exact sequence proved by $\mathrm{M}$. Auslander [1]. Before stating it, let us explain some notations. Given $A$ an $R$-module, let

$$
\cdots \rightarrow F_{n} \rightarrow F_{n-1} \rightarrow \cdots \rightarrow F_{0} \rightarrow A \rightarrow 0
$$

Received by the editors August 13, 1984.

1980 Mathematics Subject Classification. Primary 13C10, 13D05; Secondary 13H10.

Kel w'ords and phrases. Projective dimension, Ext, Tor, commutative noetherian local ring, depth, Krull dimension, artinian module. 
be a free resolution of $A$. We let $F_{-1}=A$. Set $\Omega^{0} A=A, \Omega^{p} A=\operatorname{Kernel}\left(F_{p-1} \rightarrow F_{p-2}\right)$ and $D \Omega^{p} A=\operatorname{Cokernel}\left(F_{p}{ }^{*} \rightarrow F_{p+1}{ }^{*}\right)$. Although these definitions depend on the chosen resolution of $A$, still they are well defined up to "projective equivalence." A consequence will be that the functors $\operatorname{Ext}_{R}^{i}\left(D \Omega^{p} A,\right)$ and $\operatorname{Tor}_{i}^{R}\left(D \Omega^{p} A,\right)$ are unambiguously defined, provided $i \geqslant 1$.

Proposition 1. For every pair of modules $A, B$ and every integer $p \geqslant 0$, there exists an exact sequence $\operatorname{Tor}_{2}^{R}\left(D \Omega^{p} A, B\right) \rightarrow \operatorname{Ext}_{R}^{p}(A, R) \otimes B \rightarrow \operatorname{Ext}_{R}^{p}(A, B) \rightarrow$ $\operatorname{Tor}_{1}^{R}\left(D \Omega^{p} A, B\right) \rightarrow 0$.

Proof. See [1].

Finally, we quote a theorem of S. Lichtenbaum which means that modules over regular local rings are "rigid."

Proposition 2. Let $R$ be a regular local ring and let $M, N$ be any pair of $R$-modules. If $\operatorname{Tor}_{i}^{R}(M, N)=(0)$ for some $i \geqslant 1$, then $\operatorname{Tor}_{j}^{R}(M, N)=(0)$ for all $j \geqslant i$.

Proof. See [4].

An easy consequence will be

Proposition 3. Let $M, N$ be modules over the regular local ring $R$. Assume $N \neq(0)$ and that depth $N=0$; then $\operatorname{Tor}_{1}^{R}(M, N)=(0)$ implies that $M$ is free.

ProOF. See [4].

THEOREM 1. Let $R$ be a regular local ring. A nonzero $R$-module $M$ is a strong test module, if and only if depth $M \leqslant 1$.

Proof. What remains to prove is that depth $M \leqslant 1$ implies $M$ is a strong test module. Accordingly, let $P$ be an arbitrary module satisfying $\operatorname{Ext}_{R}^{1}(P, M)=(0)$.

Krull dimension is abbreviated as $\operatorname{dim}$. If $\operatorname{dim} R=0, R$ is a field and any $R$-module is projective. Next, if $\operatorname{dim} R=1$, then p.d. $P \leqslant 1$. Lemma 1 rules out p.d. $P=1$. Hence, $P$ is projective. Hereafter, we are permitted to assume $\operatorname{dim} R \geqslant 2$. We distinguish two cases according as depth $M=0$ or depth $M=1$.

Case (i). depth $M=0$. Proposition 1 implies $\operatorname{Tor}_{1}^{R}\left(D \Omega^{1} P, M\right)=(0)$. By Proposition $3, D \Omega^{1} P$ is free. Since $\Omega^{1} P$ is projectively equivalent to $D D \Omega^{1} P, \Omega^{1} P$ must be free, i.e. p.d. $P \leqslant 1$. Referring to Lemma 1 , we conclude that $P$ is free.

Case (ii). depth $M=1$. This implies that the maximal ideal of $R$ is not associated to the zero submodule of $M$. Suppose $3_{1}, 3_{2}, \ldots, 3_{r}$ is any finite set of prime ideals each different from $m$. Then by standard arguments one can find an element $x$ in $m \backslash m^{2}$, which is outside of every one of the prime ideals $3_{1}, 3_{2}, \ldots, 3_{r}$. Taking, for $3_{1}, 3_{2}, \ldots, 3_{r}$, the associated prime ideals of the zero submodule of $M$, such an element $x$ has the properties (i) $x$ is not a zero divisor on $M$, (ii) $R / x R$ is a regular local ring. For a $R$-module $\Lambda$, denote $\bar{\Lambda}=\Lambda / x \Lambda$. We first show that $\bar{P}$ is $\bar{R}$-projective. Lemma 2 means that $\operatorname{Ext}_{R}^{1}(P, R)=(0)$ and that the natural map

$$
P^{*} \otimes M \rightarrow \operatorname{Hom}_{R}(P, M) \cdots
$$

is an isomorphism. A further consequence is that the sequence

$$
0 \rightarrow P^{*} \stackrel{x}{\rightarrow} P^{*} \rightarrow \bar{P}^{*} \rightarrow 0
$$


is exact, where the dual at the right extreme is over $\bar{R}$. This results by applying $\operatorname{Ext}_{R}(P$,$) to the exact sequence 0 \rightarrow R \stackrel{x}{\rightarrow} R \rightarrow \bar{R} \rightarrow 0$. This means, tensoring with $M$, the sequence

$$
P^{*} \otimes_{R} M \stackrel{x}{\rightarrow} P^{*} \otimes_{R} M \rightarrow \bar{P}^{*} \otimes_{R} M \rightarrow 0
$$

is exact. Next, applying $\operatorname{Ext}_{R}(P$,$) to the exact sequence 0 \rightarrow M \stackrel{x}{\rightarrow} M \rightarrow M / x M \rightarrow 0$ and making use of $\operatorname{Ext}_{R}^{1}(P, M)=(0)$, we find

$$
0 \rightarrow \operatorname{Hom}_{R}(P, M) \stackrel{x}{\rightarrow} \operatorname{Hom}_{R}(P, M) \rightarrow \operatorname{Hom}_{\bar{R}}(\bar{P}, \bar{M}) \rightarrow 0
$$

is exact. Noticing that $\bar{P}^{*} \otimes_{R} M \cong \bar{P}^{*} \otimes_{\bar{R}} \bar{M}$, we get the commutative diagram with exact rows

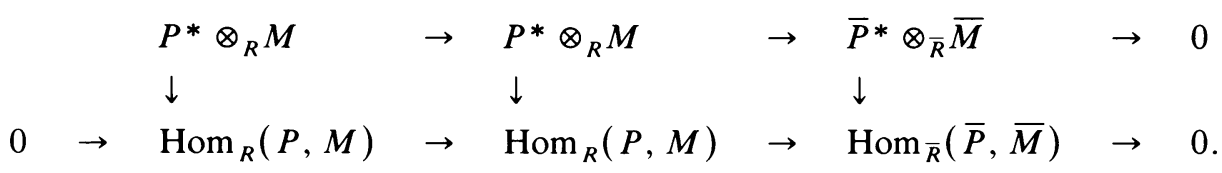

The first two vertical maps from the left are isomorphisms in view of (1). Hence by the snake lemma, the natural homomorphism

$$
\bar{P}^{*} \otimes_{\bar{R}} \bar{M} \rightarrow \operatorname{Hom}_{\bar{R}}(\bar{P}, \bar{M})
$$

is an isomorphism of $\bar{R}$-modules. Referring to Proposition 1, we conclude that $\operatorname{Tor}_{1}^{\bar{R}}(\bar{D} \bar{P}, \bar{M})=(0)$. Since $\bar{R}$ is regular local and depth $\bar{M}=0, D \bar{P}$ must be $\bar{R}$-projective by Proposition 3. From this it follows easily that $\bar{P}$ is $\bar{R}$-projective.

To complete the proof, we can certainly assume $P \neq(0)$. Now $P$ cannot be an artinian module; for if it were, then so would $\bar{P}$ be, as an $\bar{R}$-module; being free over $\bar{R}$ and $\operatorname{dim} \bar{R} \geqslant 1$, we would arrive at $\bar{P}=(0)$, i.e. $P=x P$ and so $P=(0)$, by Nakayama lemma, which would be a contradiction. Let $L$ be the maximal artinian submodule of $P$ and let $N$ be the quotient $P / L$. Note that $N \neq(0)$ and depth $N>0$. Using $\operatorname{Ext}_{R}^{1}(P, M)=(0)$ in the exact sequence $0 \rightarrow L \rightarrow P \rightarrow N \rightarrow 0$, we get a surjective homomorphism

$$
\operatorname{Hom}(L, M) \rightarrow \operatorname{Ext}_{R}^{1}(N, M) \rightarrow 0 \cdots .
$$

The data, depth $M=1, L$ is artinian immediately yields $\operatorname{Hom}(L, M)=(0)$. Hence, from (2), we find that $\operatorname{Ext}_{R}^{1}(N, M)=(0)$. Now since depth $N$ and depth $M$ are both positive, by the procedure employed in the beginning of the proof of case (ii), we can find $y \in m \backslash m^{2}$ which is not a zero divisor of both $N$ and $M$. Then starting from $\operatorname{Ext}_{R}^{1}(N, M)=(0)$, we find as before, that $N / y N$ is a projective $R / y R$-module. Now the additional information that $y$ is not a zero divisor on $N$ clearly means that $N$ is $R$-projective. Hence, the sequence, $0 \rightarrow L \rightarrow P \rightarrow N \rightarrow 0$ splits and so there is an isomorphism $P=L \oplus N$, i.e., $\bar{P}=\bar{L} \oplus \bar{N}$ (notice that $\bar{P}=P / x P$, etc.), $\bar{P}$ being $\bar{R}$-free, $\bar{L}$ is $\bar{R}$-free as well. $\bar{L}$ being a finite length $\bar{R}$-module, this is possible only when $\bar{L}=(0)$, i.e. only when $L=(0)$, by Nakayama lemma. Hence $P=N$, i.e., $P$ is free $R$-module. This finishes the proof. 
COROllary. Let $R$ be a regular local ring. Let $M$ be a nonzero $R$-module. For an arbitrary $R$-module $P$, and integer $i \geqslant 1$, the condition $\operatorname{Ext}_{R}^{i}(P, M)=(0)$ implies p. $d$. $P<i$ if and only if depth $M \leqslant 1$.

Proof. This follows from Theorem 1 by easy dimension shifting argument.

\section{REFERENCES}

1. M. Auslander, Coherent functors, Proceedings of a Conference on Categorical Algebra, La Jolla, 1965, Springer-Verlag, Berlin, Heidelberg, New York, 1966.

2. M. Auslander and O. Goldman, Maximal orders, Trans. Amer. Math. Soc. 97 (1960), 1-24.

3. P. Jothilingam, A note on grade, Nagoya Math. J. 59 (1975), 149-152.

4. S. Lichtenbaum, On the vanishing of Tor in regular local rings, Illinois J. Math. 10 (1966), 220-226.

5. M. Ramras, On the vanishing of Ext, Proc. Amer. Math. Soc. 27 (1971), 451-462.

6. J.-P. Serre, Algébre locale multiplicités, Lecture Notes in Math., vol. 11, Springer-Verlag, Berlin, 1957; 3rd. ed., 1975.

Department of Mathematics, North - Eastern Hill University, Bijni Campus, laitumkhrah, SHILLONG - 793003 (MEgHALAYA), INDIA 